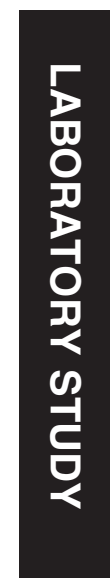

\section{A binocular iPad treatment for amblyopic children}

${ }^{1}$ Crystal Charity Ball Pediatric Vision Evaluation Center, Retina Foundation of the Southwest, Dallas, TX, USA

${ }^{2}$ Pediatric Ophthalmology and Center for Adult Strabismus, Dallas, TX, USA

${ }^{3}$ Pediatric Ophthalmology and Adult Strabismus, Plano, TX, USA

${ }^{4}$ Department of Ophthalmology, University of Texas Southwestern Medical Center, Dallas, TX, USA

Correspondence: EE Birch, Crystal Charity Ball Pediatric Vision Evaluation Center, Retina Foundation of the Southwest, 9600 North Central Expressway, Suite 200, Dallas, 75231, TX, USA Tel: +1 214363 3911; Fax: +1 2143634538 E-mail: ebirch@ retinafoundation.org

Received: 20 December 2013

Accepted in revised form: 13 June 2014

Published online: 25 July 2014

The data presented in this manuscript were presented in part, at the 2013 Annual Meeting of the Association for Research in Vision and Ophthalmology in Seattle, WA, USA.

\begin{abstract}
Purpose Monocular amblyopia treatment (patching or penalization) does not always result in $6 / 6$ vision and amblyopia often recurs. As amblyopia arises from abnormal binocular visual experience, we evaluated the effectiveness of a novel home-based binocular amblyopia treatment.
\end{abstract}

Methods Children (4-12 y) wore anaglyphic glasses to play binocular games on an iPad platform for $4 \mathrm{~h} / \mathrm{w}$ for 4 weeks. The first 25 children were assigned to sham games and then 50 children to binocular games. Children in the binocular group had the option of participating for an additional 4 weeks. Compliance was monitored with calendars and tracking fellow eye contrast settings. About half of the children in each group were also treated with patching at a different time of day. Best-corrected visual acuity, suppression, and stereoacuity were measured at baseline, at the 4- and 8-week outcome visits, and 3 months after cessation of treatment.

Results Mean ( \pm SE) visual acuity improved in the binocular group from $0.47 \pm 0.03$ $\log M A R$ at baseline to $0.39 \pm 0.03 \log M A R$ at 4 weeks $(P<0.001)$; there was no significant change for the sham group. The effect of binocular games on visual acuity did not differ for children who were patched $v s$ those who were not. The median stereoacuity remained unchanged in both groups. An additional 4 weeks of treatment did not yield additional visual acuity improvement. Visual acuity improvements were maintained for 3 months after the cessation of treatment. Conclusions Binocular iPad treatment rapidly improved visual acuity, and visual acuity was stable for at least 3 months following the cessation of treatment. Eye (2014) 28, 1246-1253; doi:10.1038/eye.2014.165; published online 25 July 2014
SL Li', RM Jost ${ }^{1}$, SE Morale ${ }^{1}$, DR Stager², L Dao ${ }^{3}$, D Stager ${ }^{3}$ and EE Birch ${ }^{1,4}$

Introduction

A growing appreciation of the role of binocular dysfunction (suppression) in amblyopia has motivated a reformulation of amblyopia treatment. Classically, amblyopia has been attributed to the habitual suppression of one eye and a reduction in cortical excitatory binocular neurons in V1, which have been thought to be established during the critical period and are lost permanently if surgical and/or optical corrections are not administered in time. ${ }^{1,2}$ However, recent evidence suggests otherwise. The loss of binocular responsiveness of V1 neurons in strabismic animals is reversible when interocular suppression is removed by using a GABA antagonist to block GABAergic inhibition. ${ }^{3}$

This suggests that the loss of binocular summation is a result of active suppression. This hypothesis received support from a report that adults with amblyopia can experience binocular vision when fellow eye contrast is reduced, revealing the presence of intact binocular cortical mechanisms. ${ }^{4,5}$ Taken together, these results suggest that it is active suppression that renders a structurally intact binocular visual system functionally monocular in amblyopia. Therefore, the structurally intact binocular pathways may be responsive to rehabilitation using binocular treatments.

Several pilot studies have reported results of a binocular treatment for amblyopia using reduced fellow eye contrast to allow the amblyopic individuals to experience binocular vision. Under investigator supervision, a total of 37 adults and 14 school-age amblyopic participants in six studies practised binocular tasks for 1-4 weeks using a haploscope, video goggles, or an iPod with a lenticular overlay to separate the monocular images. ${ }^{6-12}$ As binocular function improved with training, visual acuity also improved, usually by an average of $0.2 \log$ MAR but ranging from 0.0 to $0.9 \log$ MAR improvement. The preliminary 
studies support the hypothesis that a binocular approach to treatment of amblyopia can be efficacious. However, these initial studies were limited to small cohorts of primarily adult patients who had short-term laboratorybased treatment, and lacked long-term follow-up. Here, we report a large-cohort study that investigated the effectiveness of a home-based binocular treatment for childhood amblyopia.

\section{Materials and methods}

\section{Inclusion and exclusion criteria}

Amblyopic children aged 4-12 years with strabismus, anisometropia, or both were referred to the study by local pediatric ophthalmologists between June 2012 and September 2013. Strabismic children were only eligible to participate if misalignment of the visual axes had been successfully treated with glasses and/or surgery (defined as $\leq 5 \mathrm{pd}$ residual strabismus). Eligible children had best-corrected amblyopic eye visual acuity (BCVA) of 0.2-0.9 logMAR, 0.2 logMAR or better in the fellow eye, and $\geq 0.2 \log$ MAR interocular difference. All children enrolled had been wearing glasses (if needed) for $\geq 3$ months and had stable BCVA before baseline (three consecutive visual acuity measurements at $\geq 4$-week intervals within $\pm 0.1 \log$ MAR; for six children only two consecutive visual acuity measurements were available). Exclusion criteria were concurrent treatment with atropine penalization, prematurity $\geq 8$ weeks, developmental delay, and coexisting ocular or systemic diseases. Medical records were obtained from the referring ophthalmologists to extract diagnosis, cycloplegic refraction, and treatment plan (if any).

\section{Protocol}

The first 25 children were assigned to sham games and then 50 children to binocular games. This design was used to minimize confusion about which filter (red or green) was to be worn over which eye (right or left; amblyopic or fellow) and to allow us to conduct testing of the sham cohort with testers who were unaware that the study had a sham arm. Whether or not the child concurrently had patching treatment (at a different time of day) was decided solely by the referring pediatric ophthalmologist. Children took home the dichoptic game apps either on their own or a loaned iPad. Children wore red-green anaglyphic glasses to play the games for $4 \mathrm{~h}$ per week for 4 weeks. At the end of the first 4-week treatment period, children in the binocular group who had not attained amblyopic eye BCVA of $0.0 \log$ MAR were invited to participate in a second 4-week period. BCVA, suppression, and stereoacuity were measured at the baseline visit, the 4 - and 8-week outcome visits, and 3 months after the cessation of treatment.

\section{Binocular and sham iPad games}

Images of the dichoptic games are provided in the Supplementary Information. Each game was dichoptic, with low-contrast components visible to one eye and high-contrast components visible to the other eye. For binocular games, fellow-eye contrast was set to 15-20\% with amblyopic eye contrast $100 \%$ to allow the child to experience binocular vision. $8,9,11,12$ For the sham games, anaglyphic glasses were reversed so that amblyopic eye contrast was reduced, making it impossible for the child to experience binocular vision during game play. As the child demonstrated that they did experience binocular vision (by achieving a criterion game score), the lower contrast was gradually (5-10\%) incremented day by day. The Tetris game, which was used in four previous amblyopic treatment pilot studies, , ,9,11,12 had highcontrast falling blocks and low-contrast stationary base blocks that had to be fit together to form continuous rows of blocks. In the Balloon game, a balloon launcher was aimed to place at least three balloons of identical shape adjacent to each other, so that they 'pop' and disappear. Some balloons were high-contrast, others low-contrast, and some were visible to both eyes. Pong simulated a ping-pong game with a high-contrast ball and a lowcontrast paddle controlled by tilting the iPad from side to side. In the Labyrinth game, the child tilted the iPad to roll a high-contrast ball into a low-contrast blinking hole while avoiding other holes. At the start of each game, an anaglyphic nonius alignment cross appeared to allow the child to adjust the binocular display, if needed, for any small angle strabismus.

\section{Visual acuity}

BCVA was obtained for each eye with the E-ETDRS method. ${ }^{13}$ E-ETDRS has been validated in multiple studies and is acknowledged by the US Food and Drug Administration as a primary clinical trial endpoint. ${ }^{13-15}$

\section{Stereoacuity}

Random dot stereoacuity was evaluated using the Randot Preschool Stereoacuity Test (Stereo Optical Co., Inc., Chicago, IL, USA), the Stereo Butterfly Test (Stereo Optical Co., Inc.), and the Lang-Stereotest I (LangStereotest AG; Küsnacht, Switzerland). All stereo tests were administered and scored according to the manufacturer's instructions. 


\section{Suppression}

Severity of suppression was evaluated using a dichoptic motion coherence test developed by Mansouri et al. ${ }^{4}$ Fellow eye contrast was initially set to $0 \%$ and incremented in a 2-down-1-up staircase to determine the maximum fellow-eye contrast that still allowed the child to discriminate the direction of coherent motion. This contrast level provided a quantitative measurement of the severity of suppression.

\section{Compliance}

Each child was provided with a personalized calendar to record time spent on game play and patching, separately. 'Compliant' was defined as $>25 \%$ of prescribed hours of game play. In addition, changes in fellow-eye contrast were tracked as a secondary measure of compliance: children who did not achieve $\geq 20 \%$ increase in contrast for at least one game were classified as 'noncompliant'.

\section{Data analysis}

Effectiveness of the binocular iPad treatment for amblyopia was evaluated in an intent-to-treat analysis by paired $t$-tests for the primary BCVA outcome, and the secondary stereoacuity and suppression outcomes. BCVA improvements for different subgroups were compared with each other using one-way ANOVA and planned comparisons. As an additional amblyopia treatment, about half of the children patched the fellow eye at a different time of day than iPad game play. Therefore, in a secondary analysis, the efficacy of 4 subgroups (binocular games + patching, binocular games only, sham games + patching, and sham games only) was compared using ANOVA and planned comparisons. In another secondary analysis, efficacy of the treatment was evaluated for the subset of children with $>25 \%$ compliance with binocular iPad treatment by paired $t$-tests for BCVA, stereoacuity, and suppression. The association of number of hours of binocular iPad treatment and BCVA improvement was examined by linear regression.

\section{Statement of ethics}

We certify that all applicable institutional and governmental regulations concerning the ethical use of human volunteers were followed during this research. The study was reviewed and approved by the Institutional Review Board at University of Texas Southwestern Medical Center.

\section{Results}

Fifty amblyopic children aged 4.5-12.7 years were assigned to the binocular iPad games; five dropped from the study before the 4-week primary outcome visit because of the child's lack of interest in playing the iPad games. Twenty-five amblyopic children aged 5.7-11.9 years were assigned to sham games; one dropped before the 4-week primary outcome visit because of the child's lack of interest in playing the iPad games. Thus, primary outcome data from 45 children in the binocular group and 24 in the sham group were available for analysis.

Baseline characteristics are summarized in Table 1. In the binocular group, 10 (22\%) had strabismus, 11 (24\%) had anisometropia, and 24 (53\%) had both. At baseline, mean $( \pm \mathrm{SD}) \mathrm{BCVA}$ was $0.47 \pm 0.19 \log$ MAR and the median (range) stereoacuity was nil (nil-100 arcsec). In the sham treatment group, $5(21 \%)$ had strabismus, $11(46 \%)$ had anisometropia, and $8(33 \%)$ had both. At baseline, mean ( \pm SD) BCVA was $0.45 \pm 0.21 \log$ MAR and the median (range) stereoacuity was nil (nil-100 arcsec). Overall, 77\% had previous patching or atropine treatment for amblyopia, with a mean duration of $1.9 \pm 1.9$ years. Individual participants and their baseline characteristics are provided in Supplementary Table S1.

With an intent-to-treat analysis, the mean ( \pm SE) BCVA improved from $0.47 \pm 0.03 \log$ MAR $(6 / 18)$ at baseline to $0.39 \pm 0.03 \log$ MAR $(6 / 15)$ at 4 weeks (that is, a $0.08 \pm 0.01$ $\log$ MAR improvement; $N=45 ; t=5.84 ; P<0.001$;

Figure 1) in the binocular group but no significant BCVA improvements were found in the sham group $(N=24$; $t=1.57 ; P=0.13$ ). Twenty-three children in the binocular group and 17 children in the sham group were old enough to perform the dichoptic motion coherence task. Although the severity of suppression was reduced for most children, no significant change in the mean severity of suppression was found in either group (binocular group: $t=1.15 ; P=0.26$; sham group: $t=1.28 ; P=0.22$ ). The median (range) stereoacuity remained nil (nil-100 arcsec) at the 4-week outcome visit for both the binocular and sham groups; only five children in the binocular group $(11 \%)$ had stereoacuity improvement. Twentyseven $(60 \%)$ of the 45 children in the binocular group agreed to participate in a second 4-week binocular iPad treatment for a total of 8 weeks. However, this additional 4 weeks of treatment did not yield additional visual acuity improvements $(N=27$; paired $t=0.75 ; P=0.46)$.

As an additional amblyopia treatment, 25 children in the binocular group and 13 children in the sham group also patched the fellow eye at a different time of day than iPad game play. Thus, there were effectively four subgroups: binocular games + patching, binocular games only, sham games + patching, and sham games only. 
Table 1 Baseline characteristics of participants by group

\begin{tabular}{|c|c|c|c|c|}
\hline & \multicolumn{2}{|c|}{$\begin{array}{c}\text { Binocular } \\
(\mathrm{N}=45)\end{array}$} & \multicolumn{2}{|c|}{$\begin{array}{c}\text { Sham } \\
(\mathrm{N}=24)\end{array}$} \\
\hline & $\mathrm{n}$ & $\%$ & $\mathrm{n}$ & $\%$ \\
\hline Female & 20 & 44 & 10 & 42 \\
\hline \multicolumn{5}{|l|}{ Race/ethnicity } \\
\hline White & 39 & 87 & 19 & 79 \\
\hline Black/African American & 0 & 0 & 2 & 8 \\
\hline Hispanic & 1 & 2 & 2 & 8 \\
\hline Asian & 4 & 9 & 0 & 0 \\
\hline More than one race & 1 & 2 & 1 & 4 \\
\hline \multicolumn{5}{|l|}{ Age, yrs } \\
\hline $4-6$ & 26 & 58 & 5 & 20.8 \\
\hline $7-9$ & 16 & 36 & 18 & 75.0 \\
\hline $10-12$ & 3 & 7 & 1 & 4.2 \\
\hline \multicolumn{5}{|l|}{ Amblyopia cause } \\
\hline Strabismus & 10 & 22 & 5 & 21 \\
\hline Anisometropia & 11 & 24 & 11 & 46 \\
\hline Strabismus and ansiometropia & 24 & 53 & 8 & 33 \\
\hline \multicolumn{5}{|l|}{ BCVA amblyopic eye, $\log M A R$} \\
\hline 0.2 & 6 & 13 & 6 & 25 \\
\hline 0.3 & 7 & 16 & 4 & 17 \\
\hline 0.4 & 8 & 18 & 4 & 17 \\
\hline 0.5 & 11 & 24 & 6 & 25 \\
\hline 0.6 & 3 & 7 & 3 & 13 \\
\hline 0.7 & 6 & 13 & 1 & 4 \\
\hline 0.8 & 3 & 7 & 1 & 4 \\
\hline 0.9 & 1 & 2 & 0 & 0 \\
\hline 1.0 & 0 & 0 & 1 & 4 \\
\hline \multicolumn{5}{|l|}{ BCVA fellow eye, $\log M A R$} \\
\hline-0.1 & 15 & 33 & 7 & 29 \\
\hline 0.0 & 12 & 27 & 5 & 21 \\
\hline 0.1 & 13 & 29 & 9 & 38 \\
\hline 0.2 & 5 & 11 & 3 & 13 \\
\hline \multicolumn{5}{|l|}{ Patching during iPad study } \\
\hline Yes & 25 & 56 & 13 & 54 \\
\hline No & 20 & 44 & 11 & 46 \\
\hline
\end{tabular}

After 4 weeks, the mean ( \pm SE) BCVA improved significantly in the binocular games + patching group $(0.10 \pm 0.02 \log$ MAR; $N=25 ; t=4.52 ; P<0.01)$ and binocular games only group $(0.06 \pm 0.02 \log$ MAR; $N=20$; $t=3.96 ; P<0.01)$, with no significant difference in the amount of improvement with or without patching ( $t=1.30 ; P=0.30$ ) (Figure 2). There was no significant improvement in BCVA in the sham games + patching $(0.02 \pm 0.03 \log$ MAR; $N=13 ; t=0.76 ; P=0.46)$ or sham games only groups $(0.04 \pm 0.02 \log \mathrm{MAR} ; N=11 ; t=1.79$; $P=0.10$ ). Importantly, BCVA in the binocular games + patching group improved significantly more than the sham + patching group $(\mathrm{F}=27.77 ; P<0.01)$ and the binocular games only group improved significantly more than the sham games only group $(\mathrm{F}=13.86$; $P<0.01)$. In other words, significantly more BCVA improvement was observed when the children played binocular games with or without patching compared with sham games with or without patching.

In the binocular group, the amount of BCVA improvement was not correlated with the reported number of hours of iPad game play $(r=0.05 ; P=0.74)$, nor with the reported number of hours of patching $(r=0.04 ; P=0.81)$.

To examine efficacy of the binocular iPad treatment, a secondary analysis was conducted excluding the 11 children who had poor $(\leq 25 \%)$ compliance. As shown in Figure 3, after 4 weeks of binocular treatment, 8 (24\%) of the compliant children experienced 0.2-0.3 logMAR BCVA improvement and 17 (50\%) had 0.1 logMAR BCVA improvement. Two achieved 0.0 logMAR (6/6). Only nine $(27 \%)$ failed to experience any BCVA improvement. Statistical analyses found significant BCVA improvement among the 34 compliant children (mean $( \pm \mathrm{SE})=0.10 \pm 0.02 \log \mathrm{MAR} ; t=6.04 ; P<0.001)$ but not among the 11 non-compliant children (mean $( \pm \mathrm{SE}$ ) improvement $=0.03 \pm 0.02 \log$ MAR or 1.5 letters; $t=1.41$; $P=0.20$ ).

Among compliant children, both children who concurrently patched (at a different time of day) and children who did not patch had significant improvement in the mean $( \pm \mathrm{SE}) \mathrm{BCVA}$ at the 4-week outcome visit $(0.12 \pm 0.02 \log$ MAR, $P<0.001$ and $0.07 \pm 0.02 \log$ MAR, $P=0.001$, respectively) but the amount of BCVA improvement was not significantly different for patchers and non-patchers $(t=1.31 ; P=0.20)$.

There was no significant difference in the amount of BCVA improvement in younger $(<7 \mathrm{y})$ vs older $(\geq 7 \mathrm{y})$ children, in children with no prior amblyopia treatment $v s$ those who had prior patching or atropine treatment, in children with severe (baseline BCVA $>0.6 \log$ MAR) vs moderate amblyopia (baseline BCVA $\leq 0.6 \log \mathrm{MAR}$ ), or among children with different etiologies (strabismus, anisometropia, or both; $P \geq 0.18$ for all pairwise comparisons).

Twenty-three (68\%) of the 34 compliant children in the binocular iPad group participated in a second 4-week binocular iPad treatment for a total of 8 weeks. Figure 4 illustrates how BCVA changed over time with the binocular iPad treatment for these 23 children. The mean $\mathrm{BCVA} \pm \mathrm{SE}$ at baseline was $0.46 \pm 0.04 \log \mathrm{MAR}(6 / 17)$, which improved to $0.34 \pm 0.05 \log$ MAR $(6 / 13)$ at the 4-week treatment outcome visit; that is, an improvement of $0.12 \log$ MAR. An additional 4 weeks of treatment did not result in additional BCVA improvement (mean BVCA at 8 weeks $\pm \mathrm{SE}=0.33 \pm 0.04(6 / 13)$; paired $t=0.56$; $P=0.58)$. 


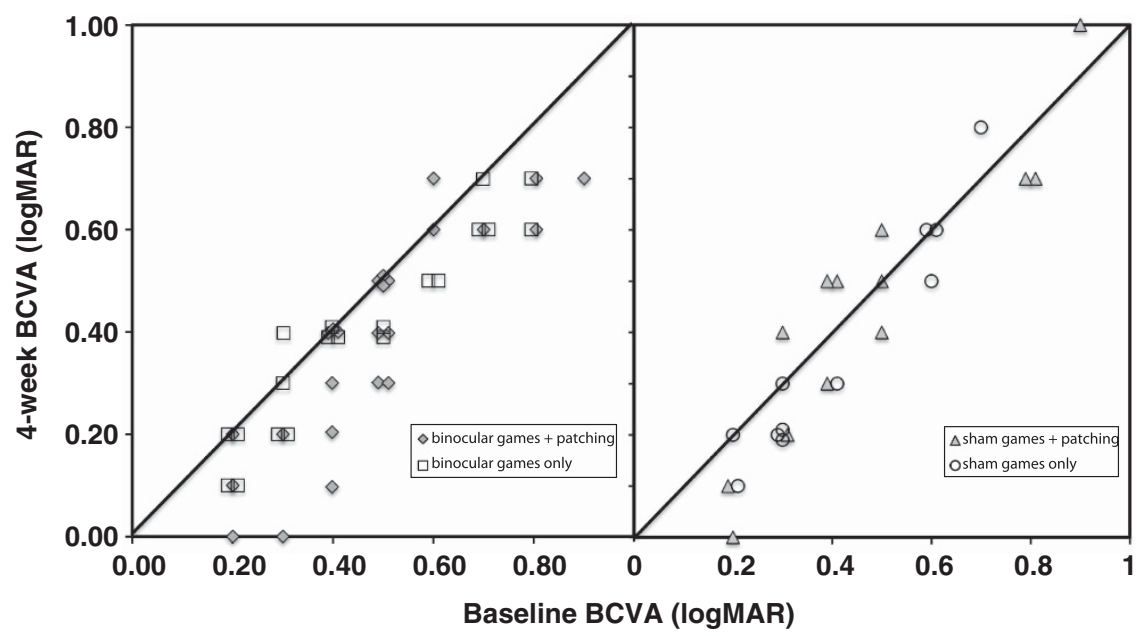

Figure 1 BCVA of the amblyopic eye at the baseline visit and the 4-week primary outcome visit. Children were assigned to either binocular games or sham games. As an additonal amblyopia treatment, about half of the children in each group also patched the fellow eye at a different time of day than iPad game play. Data points below the diagonal line represent improvement in BCVA.

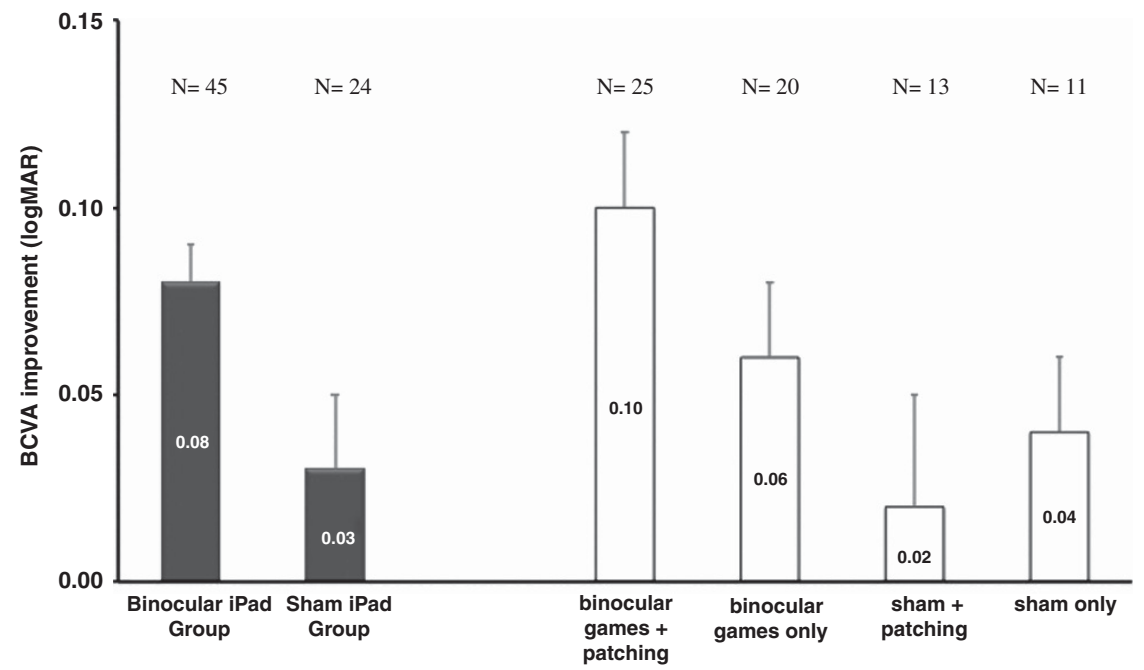

Figure 2 Change in the BCVA of the amblyopic eye after 4 weeks of game play (baseline BCVA - outcome BCVA). Left: Change in BCVA for binocular games group and sham games group. Right: As an additonal amblyopia treatment, about half of the children in both the binocular games and sham game groups also patched the fellow eye at a different time of day than iPad game play. Change in BCVA for the four subgroups is shown: binocular games + patching, binocular games only, sham games + patching, and sham games only.

Of the 34 children compliant with the first 4-week iPad treatment, 21 (62\%) completed a follow-up visit 3 months after the cessation of treatment (which lasted either 4 or 8 weeks). At 3 months post treatment, the mean BCVA did not differ significantly from the improvement measured at the final treatment outcome visit (paired $t=1.07 ; P=0.30$ ).

\section{Discussion}

Four weeks of binocular iPad game play with reduced fellow-eye contrast was an effective amblyopia treatment for 4- to 12-year-old amblyopic children, resulting in significant improvement in visual acuity. Children assigned to sham iPad games did not achieve significant improvement over the same 4 -week period. Although some children assigned to either binocular and sham games were also prescribed patching by their ophthalmologists, BCVA improved significantly only among children who played binocular games (in subgroups with or without patching), not in those who played sham games (with or without patching).

In the present study, patching was ineffective in improving visual acuity either as sole treatment (sham games + patching subgroup) and also failed to augment outcomes achieved with binocular iPad game play (binocular games + patching vs binocular games 


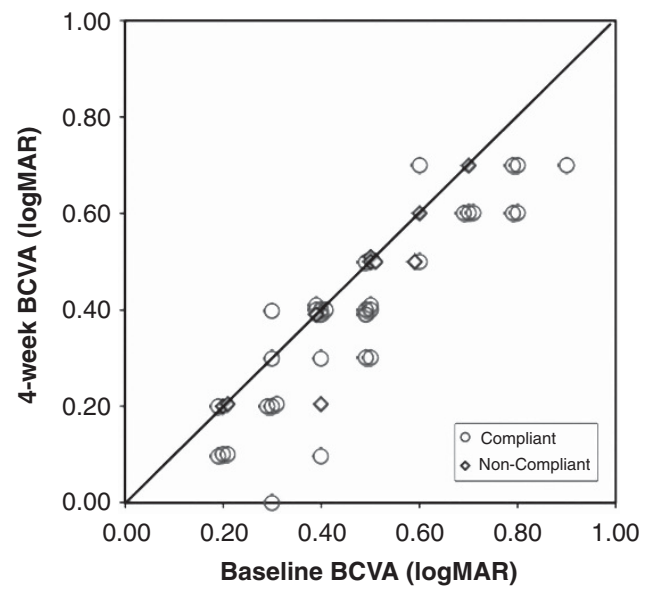

Figure 3 BCVA of the amblyopic eye at the baseline visit and the 4-week primary outcome visit for children who were compliant or non-compliant with iPad game play. Data points below the diagnonal line represent improvement in BCVA.

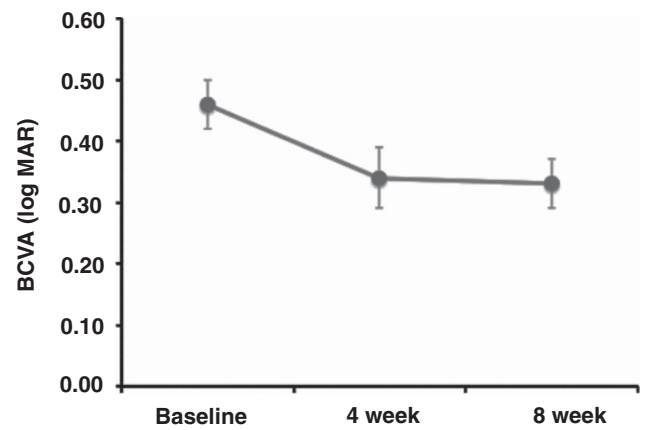

Figure 4 BCVA of the amblyopic eye at baseline, at the 4-week primary outcome visit, and after an additional 4 weeks on binocular game play (8-week outcome). Data are shown for the 23 compliant children who participated in a second 4-week period of binocular iPad game play for a total of 8 weeks.

subgroups). The failure to observe a benefit of patching may have been related to the high prevalence of prior amblyopia treatment in our cohort and the requirement for stable visual acuity at baseline; $77 \%$ had prior patching treatment lasting a mean of $1.9 \pm 1.9$ years and three visual acuity measurements at $\geq 4$-week intervals within $\pm 0.1 \log$ MAR. For a similar cohort of children, PEDIG recently reported only $0.05 \log$ MAR improvement with 12 weeks of patching treatment. ${ }^{16}$

Prior studies using reduced fellow eye contrast to allow amblyopic adults to experience binocular vision in supervised laboratory training sessions have reported more visual acuity improvements than observed in the present study (0.17-0.26 logMAR over 1-9 weeks). ${ }^{6-11}$ The greater improvements may have resulted from differences in attention or motivation due to the age of the participants and/or differences between the supervised laboratory $v s$ home setting for treatment. In the present study, $24 \%$ of the amblyopic children enrolled had poor $(\leq 25 \%)$ compliance with the binocular iPad treatment. For the adult laboratory-based studies, only data from adults who completed the study are reported. ${ }^{6-11}$ The present study and the laboratory-based studies also had significant differences in the baseline characteristics of amblyopic participants that may have affected the amount of visual acuity improvement observed. Most of the 37 participants in the laboratorybased studies had anisometropic amblyopia (54\%) and most had no prior amblyopia treatment or surgery (59\%). In the present study, only 11 (24\%) had anisometropic amblyopia and most ( $N=40$ in the binocular group; $89 \%)$ already had prior amblyopia treatment with patching or atropine.

We observed no significant change in the severity of suppression with repeated binocular game play and visual acuity improvement was not correlated with reduction in severity of suppression. Using the same method to assess severity of suppression, several laboratory studies reported a significant reduction in suppression in adults after repeated binocular game play and that the change in severity was correlated with the change in visual acuity. ${ }^{6-11}$ On the other hand, Knox et al $^{12}$ reported results similar to the present study; there was no significant reduction in suppression with binocular treatment of amblyopic schoolchildren, and, although $50 \%$ of children had some reduction in severity of suppression, there was no significant correlation with visual acuity improvement. The discrepancies between adults and children may reflect the difficulty that children encounter in making global motion coherence judgments used to quantify severity of suppression, which may have introduced more variability for children than adults. Alternatively, because all of the adult studies required repeated practice and/or testing on the dichoptic motion coherence task, ${ }^{6-11}$ the adults may have experienced perceptual learning improvements in task performance that simply coincided with their visual acuity gains.

Only $11 \%$ of children in the present study had improved random dot stereoacuity at the 4-week outcome visit. The laboratory-based studies have reported that 50-60\% achieved improved stereoacuity. ${ }^{6-12}$ This difference may, in part, be due to the different stereoacuity tests employed, including some with monocular cues. ${ }^{17-19}$ That monocular cues may underlie artifactual stereoacuity results is underscored by reports in some of the papers in which patients with tropias of 4-20 deg (8-40 pd) achieved stereoacuity of 20-500 arcsec.

Other active visual tasks have also been developed for the treatment of amblyopia. One monocular approach has been perceptual learning as an adjunct to patching for amblyopic adults, requiring practice on challenging 
visual tasks or action video games using the amblyopic eye only. ${ }^{20}$ This treatment typically results in $0.1-0.2$ $\log$ MAR visual acuity improvement after $40-50 \mathrm{~h}$ of practice or game play. An interactive binocular treatment, the I-BiT, has also been described. ${ }^{21-24}$ The I-BiT presents equal-contrast central stimuli to the amblyopic eye and peripheral stimuli to the fellow eye. A pilot study on nine children who used the I-BiT for 30 min per week for 6 weeks reported that six had 0.125 $\log$ MAR or more improvement in visual acuity but had no control group. ${ }^{23}$

Our results demonstrate that repeated binocular iPad game play significantly improved visual acuity in amblyopic children who had been wearing their spectacle correction for at least 3 months and had stable visual acuity before baseline. Moreover, the visual acuity improvement occurred rapidly and is stable for at least 3 months following the cessation of treatment. In addition to the efficacy and durability of this binocular iPad treatment, it is fun and engaging and results in better compliance than patching, at the same time imposes little risk for adverse psychosocial effects.

\section{Summary}

What was known before

- Amblyopia has been classically treated through patching the fellow eye to force use of the amblyopic eye.

- However, patching does not always result in 6/6 vision and amblyopia often recurs.

- Furthermore, patching does not promote binocular cooperation, the absence of which may underlie residual and recurrent amblyopia.

What this study adds

- Repeated binocular iPad game play significantly improved visual acuity in amblyopic children who had been wearing their spectacle correction for at least 3 months and had stable visual acuity prior to baseline.

- Moreover, the visual acuity improvement occurred rapidly, and is stable for at least 3 months following the cessation of treatment.

- In addition to the efficacy and durability of this binocular iPad treatment, it is fun and engaging and results in better compliance than patching, at the same time imposes little risk for adverse psychosocial effects.

\section{Conflict of interest}

The authors declare no conflict of interest.

\section{Acknowledgements}

This work was supported by the Fight for Sight 2013 Postdoctoral Award, National Eye Institute EY022313, the ExxonMobil Community Summer Jobs Program, and the Crystal Charity Ball (Dallas, TX).

\section{References}

1 Hubel DH, Wiesel TN. Binocular interaction in striate cortex of kittens reared with artificial squint. J Neurophysiol 1965; 28: 1041-1059.

2 Tychsen L, Wong AM, Burkhalter A. Paucity of horizontal connections for binocular vision in $\mathrm{V} 1$ of naturally strabismic macaques: cytochrome oxidase compartment specificity. J Comp Neurol 2004; 474: 261-275.

3 Sengpiel F, Jirmann KU, Vorobyov V, Eysel UT. Strabismic suppression is mediated by inhibitory interactions in the primary visual cortex. Cereb Cortex 2006; 16: 1750-1758.

4 Mansouri B, Thompson B, Hess RF. Measurement of suprathreshold binocular interactions in amblyopia. Vision Res 2008; 48: 2775-2784.

5 Baker DH, Meese TS, Mansouri B, Hess RF. Binocular summation of contrast remains intact in strabismic amblyopia. Invest Ophthalmol Vis Sci 2007; 48: 5332-5338.

6 Hess RF, Mansouri B, Thompson B. A binocular approach to treating amblyopia: antisuppression therapy. Optom Vis Sci 2010; 87: 697-704.

7 Hess RF, Mansouri B, Thompson B. A new binocular approach to the treatment of amblyopia in adults well beyond the critical period of visual development. Restor Neurol Neurosci 2010; 28: 793-802.

8 Hess RF, Thompson B, Black JM, Machara G, Zhang P, Bobier WR et al. An iPod treatment of amblyopia: an updated binocular approach. Optometry 2012; 83: 87-94.

9 To L, Thompson B, Blum JR, Maehara G, Hess RF, Cooperstock JR. A game platform for treatment of amblyopia. IEEE Trans Neural Syst Rehabil Eng 2011; 19: 280-289.

10 Goodman LK, Black JM, Phillips G, Hess RF, Thompson B. Excitatory binocular interactions in two cases of alternating strabismus. J AAPOS 2011; 15: 345-349.

11 Li J, Thompson B, Deng D, Chan LY, Yu M, Hess RF. Dichoptic training enables the adult amblyopic brain to learn. Curr Biol 2013; 23: R308-R309.

12 Knox PJ, Simmers AJ, Gray LS, Cleary M. An exploratory study: prolonged periods of binocular stimulation can provide an effective treatment for childhood amblyopia. Invest Ophthalmol Vis Sci 2012; 53: 817-824.

13 Moke PS, Turpin AH, Beck RW, Holmes JM, Repka MX, Birch EE et al. Computerized method of visual acuity testing: adaptation of the amblyopia treatment study visual acuity testing protocol. Am J Ophthalmol 2001; 132: 903-909.

14 Beck RW, Moke PS, Turpin AH, Ferris 3rd, FL, SanGiovanni JP, Johnson CA et al. A computerized method of visual acuity testing: adaptation of the early treatment of diabetic retinopathy study testing protocol. Am J Ophthalmol 2003; 135: 194-205.

15 Cotter SA, Chu RH, Chandler DL, Beck RW, Holmes JM, Rice ML et al. Reliability of the electronic early treatment diabetic retinopathy study testing protocol in children 7 to $<13$ years old. Am J Ophthalmol 2003; 136: 655-661.

16 Pediatric Eye Disease Investigator GWallace DK, Lazar EL, Holmes JM, Repka MX, Cotter SA et al. A randomized trial of increasing patching for amblyopia. Ophthalmology 2013; 120: 2270-2277.

17 Fawcett SL, Birch EE. Validity of the Titmus and Randot circles tasks in children with known binocular vision disorders. J AAPOS 2003; 7: 333-338. 
18 Leske DA, Birch EE, Holmes JM. Real depth vs randot stereotests. Am J Ophthalmol 2006; 142: 699-701.

19 Odell NV, Hatt SR, Leske DA, Adams WE, Holmes JM. The effect of induced monocular blur on measures of stereoacuity. J AAPOS 2009; 13: 136-141.

20 Levi DM. Prentice award lecture 2011: removing the brakes on plasticity in the amblyopic brain. Optom Vis Sci 2012; 89: 827-838.

21 Eastgate RM, Griffiths GD, Waddingham PE, Moody AD, Butler TK, Cobb SV et al. Modified virtual reality technology for treatment of amblyopia. Eye (Lond) 2006; 20: 370-374.

22 Waddingham PE, Butler TK, Cobb SV, Moody AD, Comaish IF, Haworth SM et al. Preliminary results from the use of the novel Interactive Binocular Treatment (I-BiT) system, in the treatment of strabismic and anisometropic amblyopia. Eye (Lond) 2006; 20: 375-378.

23 Herbison N, Cobb S, Gregson R, Ash I, Eastgate R, Purdy J et al. Interactive binocular treatment (I-BiT) for amblyopia: results of a pilot study of 3D shutter glasses system. Eye (Lond) 2013; 27: 1077-1083.

24 Foss AJ, Gregson RM, MacKeith D, Herbison N, Ash IM, Cobb SV et al. Evaluation and development of a novel binocular treatment (I-BiT) system using video clips and interactive games to improve vision in children with amblyopia ('lazy eye'): study protocol for a randomised controlled trial. Trials 2013; 14: 145.

Supplementary Information accompanies this paper on Eye website (http://www.nature.com/eye) 